\title{
Experiments on the Production of Artificial Baits.
}

\author{
By
}

\section{Frank Hughes.}

IT may be well to preface the paper on the Production of Artificial Baits by a short statement as to the way in which the experiments were taken up by $\mathrm{Mr}$. Hughes.

Members of the Association are of course aware that, through the kindness of Mr. Bayly, of Torr Grove, Plymouth, a special fund was instituted for the purpose of finding out, first of all, what it was in the bait that attracted fish, and, secondly, how the attracting matter could artificially be extracted, and applied to easily procured substances; or manufactured altogether afresh.

The papers of Mr. Bateson, published in the Journal, vol. i, No. 3, discussed, at considerable length, the manner in which fish sought their food by the action of their various senses. This work formed a logical basis for the whole inquiry of artificial bait, besides proving of great scientific value.

The question has now been taken up from a chemical point of view, and practical results sought after. This preliminary paper by Mr. Hughes-a chemist from Prof. Meldola's Laboratory-shows generally the methods he has employed in trying to produce an artificial bait from natural sources.

When several preparations had been made, a long line was set somewhere in the neighbourhood of Plymouth, the hooks being baited, not only with the newly prepared substances, but with natural baits as well, so that a proper estimate might be formed by comparison.

Briefly, it may be said, that no bait has yet been discovered which appears to act as an irrestible lure to the fishes, but at the same time a considerable amount of success has been met with, and if the 
difficulty of finding a proper medium with which to convey the attracting smell could only be got over the problem would practically be solved.-W. L. CALDERWOOD, Director.

In a previous number of the Journal Mr. Bateson has shown that the majority of the fishes which are caught by the long lines are attracted to their food entirely by smell, and it has been suggested that if the odorous material could be extracted from the bait and preserved, it would be possible to keep a supply of such bait in hand, and so be more or less independent of the supply of natural bait, which is at all times very uncertain. My first experiments were mainly in this direction, to extract and preserve the odorous matter from the bait, and in this I have been to some extent successful.

Various solvents were employed for extracting. The volatile ones, such as ether and chloroform, were used in an extracting apparatus, while in other cases the bait, \&c., was cut into small pieces and soaked in the liquid at as high a temperature as could be employed without destroying the scent. This temperature appears to be about $50^{\circ} \mathrm{C}$. Ether extracts from almost all kinds of bait an oil, varying greatly in quantity; from squid and mollusca; generally this amount is extremely small and on this account quite useless, although it has in some cases attractive properties. Pilchards and mackerel give a relatively large amount of extract, owing to their oily nature; but although these are to some extent attractive, they do not appear to contain anything like the total amount of odorous matter present in the animal. Nereis and Arenicola (as worm baits) give ethereal extracts which are attractive to some fish-turbot, rockling, pouting, \&c., but the conger is not attracted by them. Chloroform extracts nothing from mollusca, and in no case was an extract having attractive properties prepared with this solvent. Olive oil and fats do not take up the scent from any of the baits mentioned. Distillation of the baits in water, or in a current of steam, only gives a smell of cooked fish, which is utterly unattractive.

Besides extracts of the baits usually employed, I have also prepared some from coarse fish; from the intestines, livers \&c., of ling, and hake, and from the hermit crabs; but have not succeeded in obtaining anything at all attractive, and I believe that, if any efficient extract is to be prepared, it must be from squid, mackerel, or pilchard. The most attractive extracts are those prepared with glycerine, but they have the disadvantage that the solvent cannot be recovered or got rid of ; but, on the other hand, they can be kept for any length of time if some boracic or salicylic acid be dissolved in the glycerine. 
When a sufficiently attractive extract is obtained, however, it does not necessarily follow that it is of any use as a bait, since there is a great difficulty in applying anything in a liquid form. To do this, some substance is needed which will soak up enough extract to retain its odour for a sufficient time in the water, and yet be soft enough to allow of the fish getting hooked. Various substances have been tried for this purpose. Pieces of flannel and bags of buttercloth filled with pulp or cotton-wool, were soaked in the extracts, but these do not retain their smell more than a few minutes. To obviate this, I tried to thicken the extracts with gelatine, but this appears to precipitate the odorous substances, and the smell is destroyed. Slices of turnip, filled with the extract under reduced pressure, were also tried; but in this case a strong vegetable odour was developed, which rendered the substance useless. Gelatine appears at first to be just the substance required, but in order to mix the extract with the gelatine heat must be applied, and this completely destroys the smell of the extracts.

Since liquid extracts appeared to be so difficult to use as bait, I tried making a kind of mash of the bait, adding small quantities of boracic acid and glycerine, to prevent decomposition. These mixtures remain unchanged for a considerable length of time, and retain their attractive properties.

Mackerel and squid treated in this manner were most attractive to the conger in the tanks of the Laboratory, but I have not caught any fish with them from the sea, although some of the bags containing the mixtures have been sucked off the hooks. Conger will also eat pastes made with flour and these mashes; but these are of no use for fishing, as they do not remain on the hooks for a sufficient length of time. If one may judge from experiments in the Aquarium, as soon as a bait reaches the bottom it is seized by the crabs, and if it is not sufficiently tough, is pulled off the hook before the fish have time to get the scent. For this reason the medium for applying an extract, or, in fact, almost any kind of preparation must be exceedingly strong, and not easily pulled to pieces. If anything of this nature can be prepared, the applicatiou of extracts will become easy, and the whole question will be very much simplified.

I have not made any experiments with essential oils, since Mr. Bourne, when at Plymouth, tried several of these, and found that none were in any way attractive; it seems improbable that fish would be attracted by anything of that nature, since, in the natural course of events, they would never meet with such bodies.

Preserving bait by freezing has also been tried, and was moderately successful. A number of squid were cleaned and placed in an air-tight jar, a small quantity of dry boracic acid, being 


\section{EXPERIMENTS ON THE PRODUCTION OF ARTIFICIAL BAIT.}

sprinkled over them; this jar was then surrounded with a mixture of ice and salt, so that the temperature did not rise above $28^{\circ} \mathrm{F}$. At the end of about a fortnight the jar was opened, and the water which had accumulated at the bottom poured out ; the jar was again closed and kept for three weeks in ice ; the squid was then of a pink colour, but was quite fresh, and fifty hooks baited with it caught four conger, while fifty with natural bait caught only one conger and two pollack. This shows conclusively that squid can be kept for a considerable time in a frozen condition, and, if this process could be carried out on a larger scale, with refrigerating apparatus, the expense need not be very great. I intend to try if pilchards can be preserved in a similar way. This has been attempted, but the temperature was allowed to rise too high, and decomposition occurred. 